Published in final edited form as:

Cancer. 2018 January 01; 124(1): 136-144. doi:10.1002/cncr.30979.

\title{
Outcomes in Adolescents and Young Adults (AYA) with Hodgkin Lymphoma (HL) Treated on US Cooperative Group Protocols: An Adult Intergroup (E2496) and Children's Oncology Group (COG AHOD 0031) Comparative Analysis
}

\author{
Tara O. Henderson, MD, MPH ${ }^{1}$, Susan K. Parsons, MD, MRP ${ }^{2}$, Kristen Wroblewski, MS ${ }^{3}$, Lu \\ Chen, $\mathrm{PhD}^{4}$, Fangxin Hong, $\mathrm{PhD}^{5}$, Sonali Smith, $\mathbf{M D}^{6}$, Jennifer McNeer, $\mathbf{M D}^{1}$, Ranjana \\ Advani, MD ${ }^{7}$, Randy D. Gascoyne, MD $^{8}$, Louis S. Constine, MD $^{9}$, Sandra Horning, MD $^{10}$, \\ Nancy L. Bartlett, MD ${ }^{11}$, Bijal Shah, MD ${ }^{12}$, Joseph M. Connors, MD ${ }^{8}$, John Leonard, MD ${ }^{13}$, \\ Brad S. Kahl, MD ${ }^{11}$, Kara Kelly, MD ${ }^{14}$, Cindy L. Schwartz, MD, MPH ${ }^{15}$, Hongli Li, MS ${ }^{16}$, \\ Jonathan W. Friedberg, MD ${ }^{9}$, Debra L. Friedman, MD ${ }^{17}$, Leo I. Gordon, MD ${ }^{18}$, and Andrew \\ M. Evens, DO ${ }^{2}$ \\ ${ }^{1}$ Department of Pediatrics, University of Chicago, Chicago, IL \\ ${ }^{2}$ Tufts Medical Center, Tufts University School of Medicine, Boston, MA \\ ${ }^{3}$ Department of Public Health Sciences, University of Chicago, Chicago, IL \\ ${ }^{4}$ Children's Oncology Group, Acadia, CA \\ ${ }^{5}$ Department of Biostatistics, Dana-Farber Cancer Institute, Boston, MA \\ ${ }^{6}$ Department of Medicine, University of Chicago, Chicago, IL \\ ${ }^{7}$ Stanford University, Palo Alto, CA \\ ${ }^{8} \mathrm{BC}$ Cancer Agency, Vancouver, BC \\ ${ }^{9}$ Departments of Radiation Oncology and Pediatrics, University of Rochester, Rochester, NY \\ ${ }^{10}$ Genentech, San Francisco, CA \\ ${ }^{11}$ Washington University School of Medicine, St. Louis, MO \\ ${ }^{12}$ Moffitt Cancer Center, Tampa, FL \\ ${ }^{13}$ Department of Medicine, Cornell Weill School of Medicine, New York, NY \\ ${ }^{14}$ Department of Pediatrics, Roswell Park Cancer Institute, Buffalo, NY
}

\footnotetext{
Corresponding Author: Tara O. Henderson, MD, MPH, Associate Professor of Pediatrics, University of Chicago Comer Children's Hospital, 5841 S. Maryland Ave. MC 4060, Chicago, IL 60615, thenderson@ peds.bsd.uchicago.edu, phone 773-702-2501, fax 773-702-9881.

Authors Contributions:

All authors participated in designing research and analyzing data. T.O.H., S.K.P., F.H., K.W., and A.E supervised the study, drafted the manuscript with critical revision and input from all authors.

Conflicts of Interest:

No authors have declared a conflict of interest with this manuscript.

Previous Presentations: This study was presented in abstract format at the 2015 Annual American Society of Hematology Meeting (Poster) in Orlando, FL and the $201610^{\text {th }}$ International Symposium on Hodgkin Lymphoma (Oral) in Cologne, Germany.
} 
${ }^{15}$ Department of Oncology, Children's Hospital of Wisconsin, Milwaukee, WI

${ }^{16}$ Fred Hutchinson Cancer Research Center, Seattle, WA

${ }^{17}$ Department of Pediatrics, Vanderbilt University, Nashville, TN

${ }^{18}$ Northwestern University Feinberg School of Medicine, Chicago, IL

\section{Abstract}

Background-There is not a clear consensus between pediatric and adult providers in the treatment of adolescent and young adults (AYAs) with Hodgkin lymphoma (HL).

Methods-We compared the outcomes of $114 \mathrm{HL}$ patients 17-21 years treated on the adult Intergroup E2496 study, comparing failure free survival (FFS), and overall survival (OS) with 391 HL similarly aged patients (17-21 years) treated on the pediatric COG AHOD0031 study.

Results-Comparing COG and E2496 AYAs, there were no significant differences in extralymphatic disease, anemia, or hypoalbuminemia. More E2496 AYAs had stage III/IV (63\% vs. $29 \%, P<0.001)$; B symptoms (63\% vs. $27 \%, P<0.001)$; fewer had bulk (33\% vs. $77 \%$, $P<0.001$ ). More COG AYAs received radiotherapy (76\% vs. $66 \%, P=0.03$ ), though in smaller doses. E2496 AYA 5-year FFS and OS were 68\% and 89\%, respectively. COG AYA 5-year FFS and OS were $81 \%$ and $97 \%$, respectively; statistically superior compared with E2496 AYAs $(P=0.001)$. In stratified multivariable analyses, E2496 AYAs had worse FFS than COG AYAs in all strata except stage I/II patients without anemia. Propensity score analysis (based on stage, anemia, bulk disease) confirmed inferior AYA FFS for E2496 compared with COG ( $P=0.004)$. On E2496, FFS was significantly divergent across age groups in E2496 ( $P=0.005)$ with inferior outcomes for those $17-21$ vs. 22-44 years. There was no difference across age on the COG study.

Conclusions-Younger AYA HL patients appear to have better outcomes treated on a pediatric trial than similarly aged patients on an adult trial. Prospective studies examining these differences are warranted.

\section{Precis}

Adolescent and young adult (AYA) Hodgkin lymphoma (HL) patients treated on an adult clinical trial had inferior outcomes compared with older patients treated within the same study. Younger AYA HL patients appear to have better outcomes treated on a pediatric clinical trial than similarly aged patients on an adult trial.

\section{Keywords}

Hodgkin lymphoma; Adolescent and young adult oncology; Outcomes; Clinical trials; Pediatric oncology

\section{Introduction}

The National Cancer Institute (NCI) recognizes adolescents and young adults (AYAs; age at cancer diagnosis 15-39 years) with cancer as a high priority population for research. ${ }^{1}$ Advances in cure rates and participation in oncology clinical trials lags in the AYA age 
group as compared with younger children and older adults. ${ }^{2,3}$ These trends apply to Hodgkin lymphoma (HL), the most common malignancy in this age group.

Although HL is highly curable, there are debates regarding the optimal regimen based on stage, bulk, and interim metabolic response. An unexplored component of treatment approach is the potential interaction between age at diagnosis and regimen. In particular, the majority of HL patients are in the AYA age range, and there have been variable guidelines for management without clear consensus. In the FDG-PET pre-response adapted era, adult oncology providers have often approached newly diagnosed patients with four to six cycles of "ABVD" (adriamycin, bleomycin, vinblastine and dacarbazine) with radiation recommended in patients with bulk disease at presentation or in patients who do not respond sufficiently to initial chemotherapy. ${ }^{4}$ The pediatric approach is based on the Children's Oncology Group (COG) chemotherapy backbone, "ABVE-PC" (adriamycin, bleomycin, vincristine, etoposide, prednisone and cyclophosphamide) with radiation decisions based on a combination of presenting features, as well as response following 2 cycles of chemotherapy ${ }^{5-8}$ Further distinguishing pediatric and adult management, the pediatric standard of care for radiation utilizes 21 Gray (Gy) involved-field radiotherapy (IFRT) versus $36 \mathrm{~Gy}$ in adult regimens.

Prior registry data showed that HL AYA patients treated with ABVD had similar outcomes to their older counterparts. ${ }^{9}$ However, NCI Surveillance, Epidemiology and End Results (SEER) data have suggested that advances in HL survival among AYAs may be less robust compared with both older and younger populations. ${ }^{3}$ Understanding the optimal treatment approach, as well as balancing both survival rates and exposures is particularly important for AYA patients with HL, in part as they will have a large number of years of life impacted by therapy-related morbidities, such as second cancer risk due to radiation exposure at a young age. ${ }^{10}$ This, in turn, can ultimately impact survivors' lifelong health-related quality of life (HRQL) and economic productivity. ${ }^{11,12}$

We sought to examine AYA patients between the ages of 17 and 21 years (the age group of those typically eligible for both pediatric- and adult-based HL clinical trials) treated on two recent, randomized adult and pediatric North American newly diagnosed HL clinical trials, namely the intergroup trial Eastern Cooperative Oncology Group (ECOG)-ACRIN E2496 and the COG trial AHOD0031, respectively. 5,13 Our aim was to compare both exposures and outcomes of different age cohorts within E2496 as well as across the two studies.

\section{Methods}

All patients provided informed consent according to federal and institutional guidelines and in accordance with the Declaration of Helsinki. The NCI-approved phase III clinical trials included in this analysis (AHOD 0031 and E2496) were conducted using IRB-approved protocols.

\section{Eligibility and Treatment}

E2496—Eligibility for E2496 included classical HL (cHL) patients with previously untreated, advanced-stage (III/IV) disease or local disease with bulky mediastinum. ${ }^{13}$ Bulk 
disease was defined by a mass over one-third the maximum intrathoracic diameter on a standing posterior-anterior chest $\mathrm{x}$-ray (peripheral bulk was not included in the definition). Histology was determined using central review when available, then local review. Between April 1999 and June 2006, 854 patients were enrolled, 794 were deemed eligible. Of those eligible patients, 114 were 17 to 21 years, 529 were 22 to 44 years, and 150 were 45 years or older.

Eligible patients were randomized to receive ABVD or Stanford V. ${ }^{13}$ ABVD was given for 6 or 8 cycles (28 day cycles), depending on response by CT scan. Stanford V chemotherapy (doxorubicin, vinblastine, nitrogen mustard, etoposide, vincristine, bleomycin, prednisone) was administered for 12 weeks. Radiation therapy (RT) was delivered to all patients with bulky mediastinal adenopathy, scheduled to begin 2 weeks after completion of chemotherapy. A dose of 36 Gy was delivered to mediastinal, bilateral hilar and bilateral supraclavicular areas. For patients who received Stanford V, 36 Gy was delivered to any pretreatment site of $>5 \mathrm{~cm}$ and for macroscopic splenic disease, as determined by CT scan.

AHOD0031-Eligible patients for AHOD0031 included patients younger than 22 years with newly diagnosed, biopsy-proven cHL, Ann Arbor stages IB, IA extra-nodal (E), IIB, IIAE, IIIA, IVA with or without bulk disease, and IA or IIA with bulk disease. ${ }^{5}$ Bulk disease was defined by: mediastinal mass with a diameter greater than one third of the thoracic diameter on an upright posterior-anterior chest $x$-ray or extramediastinal nodal aggregate $>6$ $\mathrm{cm}$ in the longest transverse diameter on axial CT scan. Between September 2002 and July 2009, 1,734 patients were enrolled and 1,712 were deemed eligible. Of those eligible, 391 patients were between 17 and 21 years.

All patients received two 21-day cycles of ABVE-PC chemotherapy, followed by CT scan response evaluation. Rapid early responders (RERs), defined as those patients who have a complete or very good response after 2 cycles of chemotherapy by CT scan, received two additional ABVE-PC cycles followed by complete response (CR) evaluation. RERs with CR were randomly assigned to undergo IFRT or observation; RERs with less than CR were nonrandomly assigned to receive IFRT. Slow-early responders (SERs) were randomly assigned to receive or not receive two cycles of dexamethasone, etoposide, cisplatin, and cytarabine (DECA) followed by two ABVE-PC cycles. All SERs were assigned to receive IFRT. All chemotherapy cycles were delivered with granulocyte colony-stimulating factor support. Patients who underwent IFRT received $21 \mathrm{~Gy}$, which was delivered 4 weeks after completion of chemotherapy to involved areas of disease at presentation. Gross tumor volume included any lymph nodes greater than $1.5 \mathrm{~cm}$ in a single axis on CT scan. Clinical target volume included the anatomic compartment containing involved nodes. The planning target volume was determined by the addition of a $1.0-\mathrm{cm}$ margin around the clinical target volume to account for patient motion and set-up variability, with allowable modification at the discretion of the treating radiation oncologist to avoid healthy tissue. In patients assigned to IFRT, patients with pulmonary metastases received 10.5 Gy of whole-lung radiation, regardless of resolution of pulmonary lesions, and those with liver metastases received 15 Gy to the entire liver. 


\section{Statistical Analysis}

We compared baseline demographics and clinical features, and planned treatment of 114 newly diagnosed ECOG-ACRIN AYA HLpatients between the ages of 17-21 years with the 391 newly diagnosed AYA HL patients between ages 17-21 years treated on the COG AHOD0031, using chi-square tests or Fisher's exact tests for categorical variables and t-tests and Wilcoxon rank-sum tests for continuous or ordinal variables. Unstratified and stratified log-rank tests, as well as propensity score analysis were utilized to compare differences in patient outcomes (FFS and OS). The initial propensity score was calculated as the predicted probability from a logistic regression model with cohort (ECOG-ACRIN E2496 vs. COG AHOD0031) as the dependent variable and stage, anemia, and bulk disease as the independent variables. These independent variables were chosen from the International Prognostic Score. ${ }^{14}$ Survival was then compared between groups using a stratified log-rank test, with quintiles of the propensity score as the stratification factor. The robustness of the results was confirmed using Cox regression with the propensity score as a covariate. A second propensity score was calculated that also incorporated age, gender, B symptoms, and albumin. C-statistics for the two propensity score models are reported.

In addition, we examined characteristics and outcomes of the 114 newly diagnosed AYA HL patients 17-21 years treated on E2496 and compared the failure free survival (FFS) and overall survival (OS) with patients ages >21 years on E2496 using log-rank tests stratified on extent of disease (stage), treatment regimen (ABVD vs. Stanford V), number of risk factors (0-2 vs. 3-7). ${ }^{15}$ Likewise, we examined characteristics and outcomes of the 391 newly diagnosed AYA HL patients 17-21 treated on COG AHOD 0031 and compared the failure free survival (FFS) and overall survival (OS) with patients ages $<17$ years on COG AHOD 0031

\section{Results}

\section{Presenting features of ECOG-ACRIN E2496 and COG AHOD0031 AYA patients}

Table 1 depicts the demographic and presenting features of the 17 to 21 year olds treated on E2496 and AHOD0031. There were no statistically significant differences in gender or race/ ethnicity between the 17-21 year olds on the two studies. AYAs on the AHOD0031 study had a mean age at enrollment that was younger than those on E2496 (18.2 years vs. 20.1 years; $P<0.001)$. In terms of presenting features, there were no significant differences in histology distribution, extralymphatic disease, anemia and albumin $<4$ gram $/ \mathrm{dL}$. A larger proportion of ECOG-ACRIN AYAs were stage III and IV (63\% vs. $29 \%, P<0.001)$. A significantly larger proportion of ECOG-ACRIN AYAs had B symptoms (63\% vs. 27\%, $P<0.001$ ), while a significantly smaller proportion of E2496 AYAs had mediastinal bulk disease at presentation $(33 \%$ vs. $77 \%, P<0.001)$.

\section{Comparison of planned cumulative chemotherapy and radiation exposures}

Cumulative planned doses of the chemotherapy drugs administered in E2496 and COG AHOD0031 are depicted in Table 2. Of the three regimens, ABVE-PC, Stanford V, and ABVD, E2496 patients treated with 6 to 8 cycles of ABVD received the highest cumulative doses of anthracyclines, bleomycin and alkylator chemotherapy. However, these patients 
received no corticosteroids or etoposide. Both E2496 patients treated with Stanford V and AHOD0031 patients received etoposide, though AHOD0031 patients received more of this exposure. SERs in the AHOD0031 study randomized to DECA chemotherapy ( $\mathrm{N}=36$ AYA patients) were also exposed to cytarabine and cisplatin, though in relatively low cumulative exposures. A greater proportion of COG AYA's received radiotherapy ( $76.2 \%$ vs. $65.8 \%)$ though in lower doses, (21 Gy vs. $36 \mathrm{~Gy}$ ).

\section{Outcomes of E2496 AYAs compared with COG AHOD0031 AYAs}

For survival comparison across studies, COG AYAs had statistically superior FFS compared with E2496 AYAs ( $P=0.001)$, see Figure 1. More COG AYAs received radiotherapy (76\% vs. $66 \%, P=0.03$ ), though in smaller doses (21Gy vs. 36Gy). In each group, 3 AYAs developed a second cancer.

In survival analyses stratified by stage and anemia, E2496 AYAs had worse FFS compared with COG AYAs in all strata except among the subgroup of patients with stage I/II without anemia (data not shown). Furthermore, propensity score analysis matched on stage, anemia, and bulk disease (c-statistic $=0.75$ ) confirmed the inferior FFS for E2496 AYAs compared with COG AYAs $(P=0.004)$. Moreover, the AYA survival disparity across studies persisted after additional covariates were incorporated into the propensity score (i.e., age, gender, B symptoms, and hypoalbuminemia with a c-statistic $=0.90 ; P=0.026$, see Supplementary Material).

\section{E2496 AYA outcomes vs. older E2496 patients}

In E2496, the 5-year FFS and OS rates for AYAs between the ages of 17 and 21 years were $68 \%$ and $89 \%$, respectively. There were significantly divergent outcomes in FFS and OS by patient age (see Table 3). There was no FFS difference between AYAs treated with ABVD vs. Stanford V regimens ( $P=0.66$ ). However, FFS was significantly different across age groups in E2496 ( $P=0.005$, see Figure 1A and 1C). The difference was driven by older patients (age $>=60$ ), and also suggested AYAs may have inferior outcomes to patients diagnosed between 22 and 44 years of age. Interestingly, AYA FFS outcomes on E2496 appeared more similar to patients $45-59$ years.

\section{COG AHOD0031 AYA outcomes vs younger AHOD 0031 patients}

The 5-year FFS and OS for AYAs on the COG study were $81 \%$ and $97 \%$, respectively (see Figure 1B and 2D). In contrast to the E2496 study, there were no statistical differences in either FFS or OS between those 17 and 21 years and those younger than 17 years treated on the COG study ( $P=0.11$ and $P=0.77$, respectively).

\section{Discussion}

In 2006, the NCI and Livestrong Foundation published a Progress Review Group (PRG) entitled "Closing the Gap: Research and Care Imperatives for Adolescents and Young Adults with Cancer" that recommended that a more robust research effort to better understand specific cancer outcomes in AYAs and the factors associated with these outcomes. ${ }^{1}$ Responsive to the PRG, this is the first large retrospective analysis of AYA HL patients 
treated on recent North American clinical trials. AYA HL patients between the ages of 17 and 21 years treated on E2496 had inferior outcomes compared with older patients (i.e., ages 22 to 44 years) treated within the same study, as well as to similarly matched AYA patients treated on the COG study, AHOD0031.

A prior retrospective registry analyses from the British Columbia Cancer Agency (BCCA) did not observe differences between AYAs and older adults who were treated with ABVD. ${ }^{9}, 16$ The discrepant results of the BC data versus the current analysis of inferior outcomes in 17-21 year olds in the E2496 study (compared both to older patients on the same study and to COG AYA outcomes) are not clear, several potential explanations exist. First, methodological variances or clinical and demographic differences may have been factors, including trial design differences; indeed, there was a larger proportion of E2496 patients with stage III or IV with bulk disease (see Table I footnote). Moreover, the definitions of bulk between the COG and ECOG-ACRIN groups were different (in addition to mediastinal mass with a diameter $>1 / 3$ of the thoracic diameter on chest $\mathrm{x}$-ray, COG included patients with extramediastinal nodal aggregate $>6 \mathrm{~cm}$ in the longest transverse diameter on axial CT scan while ECOG-ACRIN did not). However, analyses controlling for stage at diagnosis, presence of B symptoms or bulk disease, as well as other prognostic factors, still reflected these worse outcomes in E2496 AYAs. Additionally, while the aforementioned BCCA study was a retrospective registry analysis with strength of complete data in a centralized care setting, the current data have the strength of being derived from prospective, randomized, controlled clinical trials.

Clinical practices for AYAs receiving treatment in pediatric versus adult centers may influence patient outcomes such as treatment regimen differences or dose-intensity of the treatment plans. General compliance differences of AYA patients treated at a pediatric vs. adult center may have played a role. In an analysis of AYAs with acute lymphoblastic leukemia, ages 16-20 years, investigators in fact found that those treated on adult clinical trials had outcomes inferior to those treated on pediatric trials and they hypothesized that in part, adherence and lack of support services contributed to poorer outcomes on the adult trials. ${ }^{17}$ Psychosocial support and related services are typically more available and comprehensive at pediatric cancer centers in the US. Furthermore, the COG regimen, $\mathrm{ABVE}-\mathrm{PC}$, is a dose intense regimen given every three weeks. While delivered in lower doses, more COG AYAs received radiotherapy. Both in AHOD0031 and in the original study of ABVE-PC, there were minimal treatment delays or dose modifications (personal communication, Friedman). ${ }^{6}$ In comparison, investigators were given specific directives to dose delay and modify ABVD therapy for modest cytopenias in the E2496 trial (e.g., doxorubicin and vinblastine decreased $25 \%$ for absolute neutrophil count (ANC) $<1,800$; and all drugs held for at least 1 week with ANC <1,200). Unfortunately, dose delays and modifications were not captured as part of data collection in E2496. More recent US intergroup studies using the ABVD backbone do not allow for delay or dose reduction for neutropenia and future comparison between patients treated on these adult cooperative group studies with those receiving pediatric regimens may better elucidate differences in the efficacy of chemotherapy backbones. 
Disease biology for AYAs as compared to older counterparts could possibly explain these differences. A COG group recently reported the application of a published gene expression profiling 23-gene model developed for adults in 185 pediatric AHOD 0031 patients. The assay failed to predict outcomes, suggesting that distinct biology predicts treatment failure in pediatric patients, ${ }^{18}$ though this study did not take into account the different treatment regimens between pediatric and adult providers. Also suggestive of distinct biologic features of AYA patients, a sub-group analysis of a recently completed phase II Southwestern Oncology Group (SWOG) S0816 US Intergroup clinical trial of stage III/IV HL patients treated with response-adapted ABVD (i.e., including augmented therapy with BEACOPP in patients who were PET2 positive) suggested that younger AYAs may have inferior outcomes to their older counterparts. The 3-year FFS for AYAs ages 18-21 years $(\mathrm{N}=40)$ was $70 \%$ (95\% CI: $52.8 \%, 81.5 \%$ ) as compared to those $22-44$ was $77 \%$ (N=226; 95\% CI: $71.0 \%$, $82.2 \%$ ), while those $45-59$ was $83 \%$ (N=68; 95\% CI: 72.0\%, 90.5\%) (see Supplementary Figure for Survival Curves).${ }^{19}$ It is important to highlight that in addition to response adapted therapy as noted above, ABVD was prescribed in S0816 to be given in full doses and without treatment delay, irrespective of the ANC on day of treatment, as has been previously published. ${ }^{20,21}$

Collectively, when considering the treatment of young patients with HL, it is also imperative to consider therapeutic exposures. HL is associated with high rates of premature mortality due to significant therapy-related morbidities, including cardiac disease, pulmonary disease and second cancers. Studies examining the late effects of childhood cancer and more specifically, HL, have demonstrated that these late effects and second cancers are related to specific exposures such as cumulative exposure to anthracyclines, bleomycin and alkylators, as well as radiation dose and field. In our analysis, patients treated according to the COG protocol received $200 \mathrm{mg} / \mathrm{m}^{2}$ doxorubicin, $3.2 \mathrm{~g} / \mathrm{m}^{2}$ alkylator (cyclophosphamide), $60 \mathrm{U} / \mathrm{m}^{2}$ bleomycin, $11.2 \mathrm{mg} / \mathrm{m} 2$ vincristine, $1,500 \mathrm{mg} / \mathrm{m}^{2}$ etoposide and prednisone. A small subset of patients who were SER to initial therapy received an additional $400 \mathrm{mg} / \mathrm{m}^{2}$ etoposide, dexamethasone, cytarabine $12 \mathrm{grams} / \mathrm{m}^{2}$, and cisplatin $180 \mathrm{mg} / \mathrm{m}^{2}$. Seventy-six percent of pediatric patients received RT. In comparison, ABVD patients received higher doses of doxorubicin (300-400mg/ $\mathrm{m}^{2}$, reflecting 6 or 8 cycles), bleomycin $\left(120-180 \mathrm{U} / \mathrm{m}^{2}\right)$, and dacarbazine chemotherapy $\left(4.5-6.0 \mathrm{grams} / \mathrm{m}^{2}\right)$. In general, chemotherapeutic exposures in E2496 patients who received Stanford V chemotherapy were lower than in the ABVD patients. Stanford V exposure included doxorubicin $150 \mathrm{mg} / \mathrm{m}^{2}$, vinblastine $36 \mathrm{mg} / \mathrm{m}^{2}$, nitrogen mustard $18 \mathrm{mg} / \mathrm{m}^{2}$, etoposide $180 \mathrm{mg} / \mathrm{m}^{2}$, vincristine $8.4 \mathrm{mg} / \mathrm{m}^{2}$, bleomycin 60 $\mathrm{U} / \mathrm{m}^{2}$. However, planned RT was more extensive in patients randomized to Stanford V. Overall, fewer patients received RT in the E2496 study ( 66\%). While exposures differed, the absolute number of second cancers reported in each study was the same $(\mathrm{N}=3)$, although longer follow-up is warranted. Unfortunately, the late cardiac and pulmonary outcomes have not been examined to date in either of these trials.

Our study has several limitations that need to be considered when interpreting these results. This was not a randomized comparison of ECOG-ACRIN and COG treatment approaches in AYAs. As such, there were inherent trial design and patient characteristic differences between E2496 and AHOD0031 that limit our ability to draw definitive conclusions. The 
staging evaluation and response criteria were not uniform across studies and unfortunately, and despite harmonization efforts being undertaken during the last two decades (International Harmonization Project and the Lugano Classification) it is very difficult to compare between trials. ${ }^{22,23}$ We recognize that while we utilized propensity scores to take into account these differences, this methodology alone cannot guarantee that these two cohorts are comparable. Moreover, our ability to examine adherence to the prescribed treatment in each of the trials was limited in that providers were at their own discretion in determining delays and dose modifications and documentation of these delays and modifications were not mandated by the trial.

In conclusion, the results of our retrospective analysis of two recent phase III randomized HL studies showed that younger AYAs (aged 17 to 21 years) treated on the Intergroup trial E2496 had inferior outcomes to older patients treated on the same study and to similarly aged patients treated on a pediatric trial, COG AHOD0031. These outcomes may result from treatment regimen differences or dose-intensity, treatment compliance, differing patient populations or risk profiles, disease biology and/or other factors. Prospective examination of these issues in AYA HL patients is needed.

\section{Supplementary Material}

Refer to Web version on PubMed Central for supplementary material.

\section{Acknowledgments}

Funding:

AHOD0031 was coordinated by the Children's Oncology Group and is supported by NIH grants no. U10 CA098543 and U10 CA098413.

E2496 was coordinated by ECOG-ACRIN and supported in part by Public Health Service Grants CA180816, CA180799, CA180820 and CA180794 and from the NCI, NIH and the Department of Health and Human Services.

The statistical analysis provided by Ms. Wroblewski was supported by a Leukemia and Lymphoma Society grant (Henderson/Parsons, Co-Principal Investigators).

\section{References}

1. [accessed May 30, 2012] Closing the Gap: Research and Care Imperatives for Adolescents and Young Adults with Cancer. Report of the Adolescent and Young Adult Oncology Progress Review Group. Available from URL: http://planning.cancer.gov/library/

AYAO_PRG_Report_2006_FINAL.pdf

2. Bleyer WA. Cancer in older adolescents and young adults: epidemiology, diagnosis, treatment, survival, and importance of clinical trials. Med Pediatr Oncol. 2002; 38:1-10. [PubMed: 11835231]

3. Bleyer A, Budd T, Montello M. Adolescents and young adults with cancer: the scope of the problem and criticality of clinical trials. Cancer. 2006; 107:1645-1655. [PubMed: 16906507]

4. National Comprehensive Cancer Network (NCCN): NCCN Clinical Practice Guidelines in Oncology: Hodgkin Lymphoma. 2014

5. Friedman DL, Chen L, Wolden S, et al. Dose-intensive response-based chemotherapy and radiation therapy for children and adolescents with newly diagnosed intermediate-risk hodgkin lymphoma: a report from the Children's Oncology Group Study AHOD0031. J Clin Oncol. 2014; 32:3651-3658. [PubMed: 25311218] 
6. Schwartz CL, Constine LS, Villaluna D, et al. A risk-adapted, response-based approach using ABVE-PC for children and adolescents with intermediate- and high-risk Hodgkin lymphoma: the results of P9425. Blood. 2009; 114:2051-2059. [PubMed: 19584400]

7. Kelly KM, Hodgson D, Appel B, et al. Children's Oncology Group's 2013 blueprint for research: Hodgkin lymphoma. Pediatr Blood Cancer. 2013; 60:972-978. [PubMed: 23255501]

8. Mauz-Korholz C, Metzger ML, Kelly KM, et al. Pediatric Hodgkin Lymphoma. J Clin Oncol. 2015; 33:2975-2985. [PubMed: 26304892]

9. Foltz LM, Song KW, Connors JM. Hodgkin's lymphoma in adolescents. J Clin Oncol. 2006; 24:2520-2526. [PubMed: 16735704]

10. Castellino SM, Geiger AM, Mertens AC, et al. Morbidity and mortality in long-term survivors of Hodgkin lymphoma: a report from the Childhood Cancer Survivor Study. Blood. 2011; 117:18061816. [PubMed: 21037086]

11. Glimelius I, Ekberg S, Linderoth J, et al. Sick leave and disability pension in Hodgkin lymphoma survivors by stage, treatment, and follow-up time--a population-based comparative study. J Cancer Surviv. 2015; 9:599-609. [PubMed: 25689964]

12. Hanly P, Soerjomataram I, Sharp L. Measuring the societal burden of cancer: the cost of lost productivity due to premature cancer-related mortality in Europe. Int J Cancer. 2015; 136:E136145. [PubMed: 25066804]

13. Gordon LI, Hong F, Fisher RI, et al. Randomized phase III trial of ABVD versus Stanford V with or without radiation therapy in locally extensive and advanced-stage Hodgkin lymphoma: an intergroup study coordinated by the Eastern Cooperative Oncology Group (E2496). J Clin Oncol. 2013; 31:684-691. [PubMed: 23182987]

14. Diefenbach CS, Li H, Hong F, et al. Evaluation of the International Prognostic Score (IPS-7) and a Simpler Prognostic Score (IPS-3) for advanced Hodgkin lymphoma in the modern era. Br J Haematol. 2015; 171:530-538. [PubMed: 26343802]

15. Hasenclever D, Diehl V. A prognostic score for advanced Hodgkin's disease. International Prognostic Factors Project on Advanced Hodgkin's Disease. N Engl J Med. 1998; 339:1506-1514. [PubMed: 9819449]

16. Marr, KCCJ., Goddard, KJ., Savage, KJ., Deyell, RJ. American Society of Hematology. Orlando, FL: Blood; 2015. ABVD Chemotherapy Results in Excellent Outcomes with Reduced Radiation Therapy Rates in Children, Adolescents, and Young Adults with Hodgkin Lymphoma.

17. Stock W, La M, Sanford B, et al. What determines the outcomes for adolescents and young adults with acute lymphoblastic leukemia treated on cooperative group protocols? A comparison of Children's Cancer Group and Cancer and Leukemia Group B studies. Blood. 2008; 112:16461654. [PubMed: 18502832]

18. Mottok, AJRL., Chun Chan, F., Scott, DW., Friedman, D., Schwartz, CL., Kelly, K., Horton, TM., Steidl, C. 10th International Symposium on Hodgkin Lymphoma. Cologne, Germany: Haematologica; 2016. PREDICTION OF PRIMARY TREATMENT RESPONSE AND OUTCOME IN PEDIATRIC HODGKIN LYMPHOMA USING DIGITAL GENE EXPRESSION PROFILING, P069.

19. Friedberg JWLH, Schoder H, Straus D, Moskowitz C, LeBlanc M, Rimsza LM, Bartlett NL, Evens AM, Mittra ES, LaCasce A, Sweetenham J, Barr PM, Fanale MA, Knopp MV, Noy A, His ED, Cook JR, Lechowicz MJ, Gascoyne RD, Leonard JP, Kahl BS, Cheson BD, Fisher RI, Press OW. Long-term follow-up of SWOG S0816: Response adapted therapy of advanced stage Hodgkin lymphoma using early interim FDG-PET imaging. Haematologica. 2016; 101

20. Evens AM, Cilley J, Ortiz T, et al. G-CSF is not necessary to maintain over $99 \%$ dose-intensity with ABVD in the treatment of Hodgkin lymphoma: low toxicity and excellent outcomes in a 10year analysis. Br J Haematol. 2007; 137:545-552. [PubMed: 17459049]

21. Boleti E, Mead GM. ABVD for Hodgkin's lymphoma: full-dose chemotherapy without dose reductions or growth factors. Ann Oncol. 2007; 18:376-380. [PubMed: 17071938]

22. Flerlage JE, Kelly KM, Beishuizen A, et al. Staging Evaluation and Response Criteria Harmonization (SEARCH) for Childhood, Adolescent and Young Adult Hodgkin Lymphoma (CAYAHL): Methodology statement. Pediatr Blood Cancer. 2017 
23. Cheson BD. The International Harmonization Project for response criteria in lymphoma clinical trials. Hematol Oncol Clin North Am. 2007; 21:841-854. [PubMed: 17908623] 


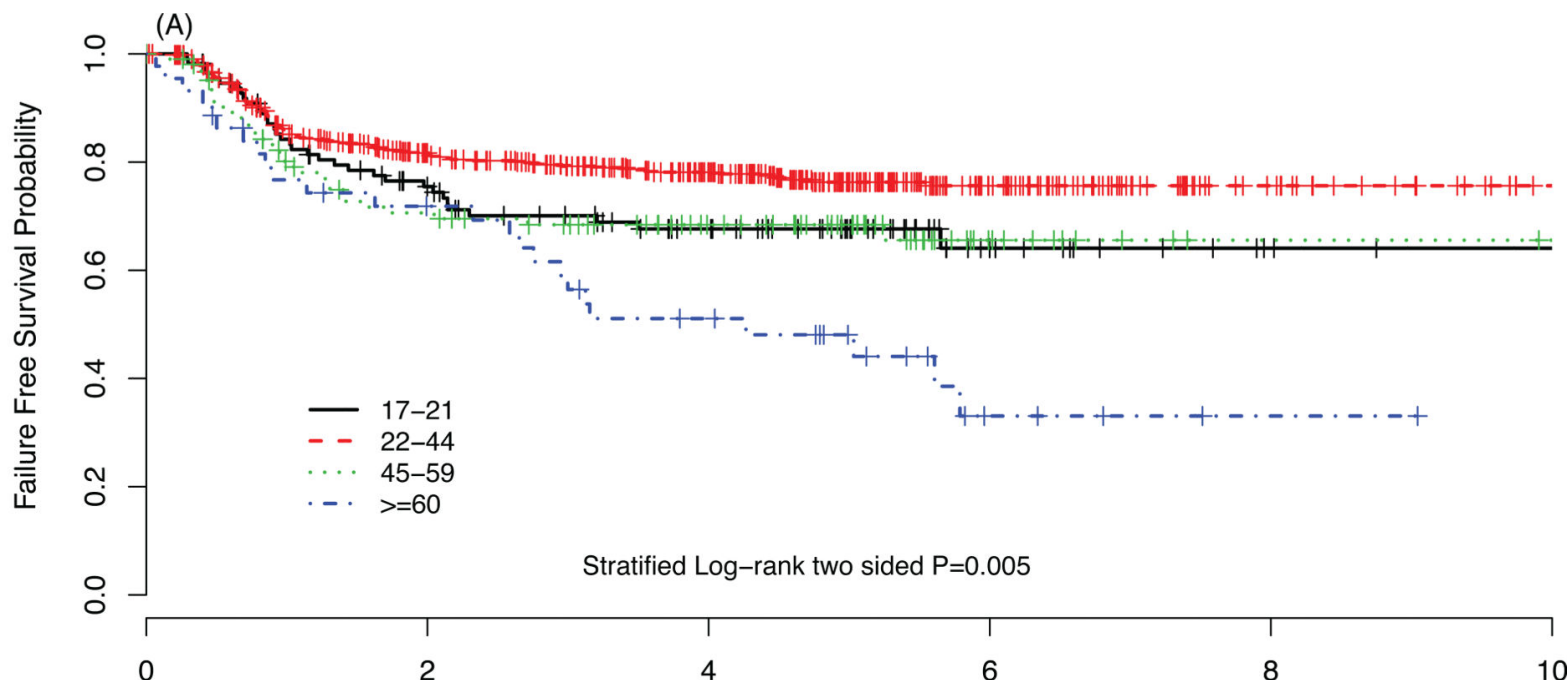

No. at Risk

$\begin{array}{rr}17-21 & 114 \\ 22-44 & 529 \\ 45-59 & 106 \\ >=60 & 44\end{array}$

72
347
66
28

Years since registration 


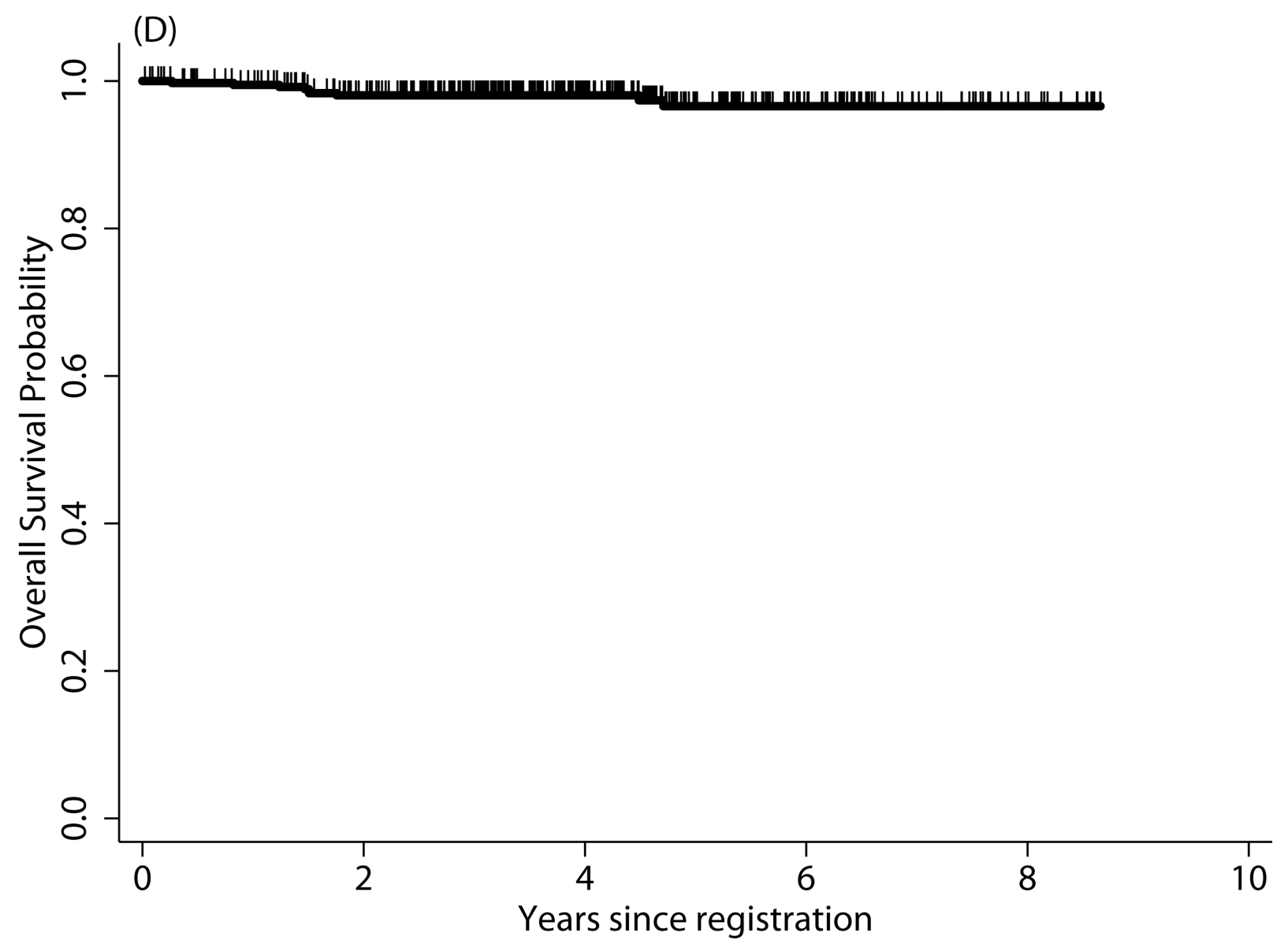

No. at Risk 
(C)

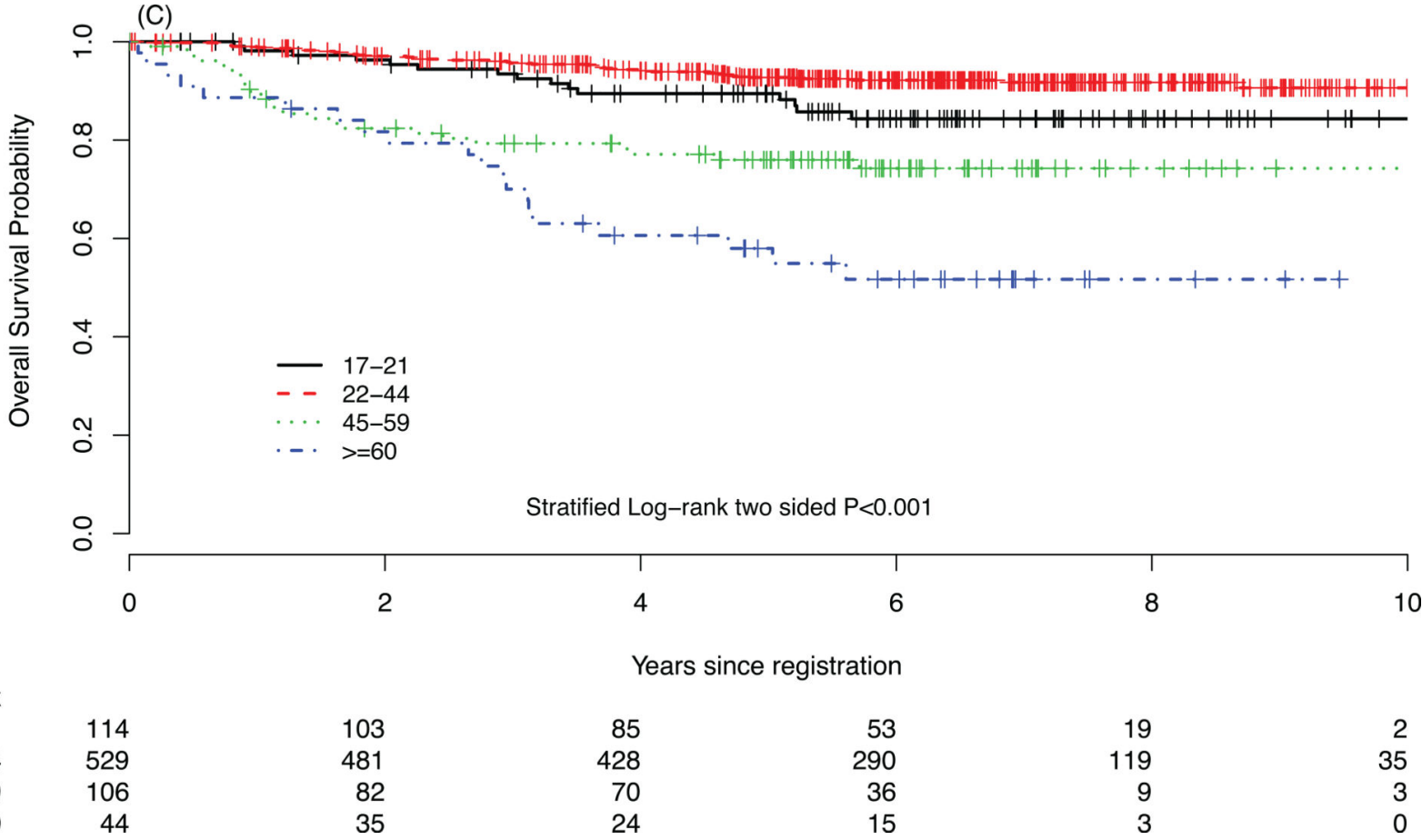

No. at Risk

$\begin{array}{rr}17-21 & 114 \\ 22-44 & 529 \\ 45-59 & 106 \\ >=60 & 44\end{array}$

35

24

15 


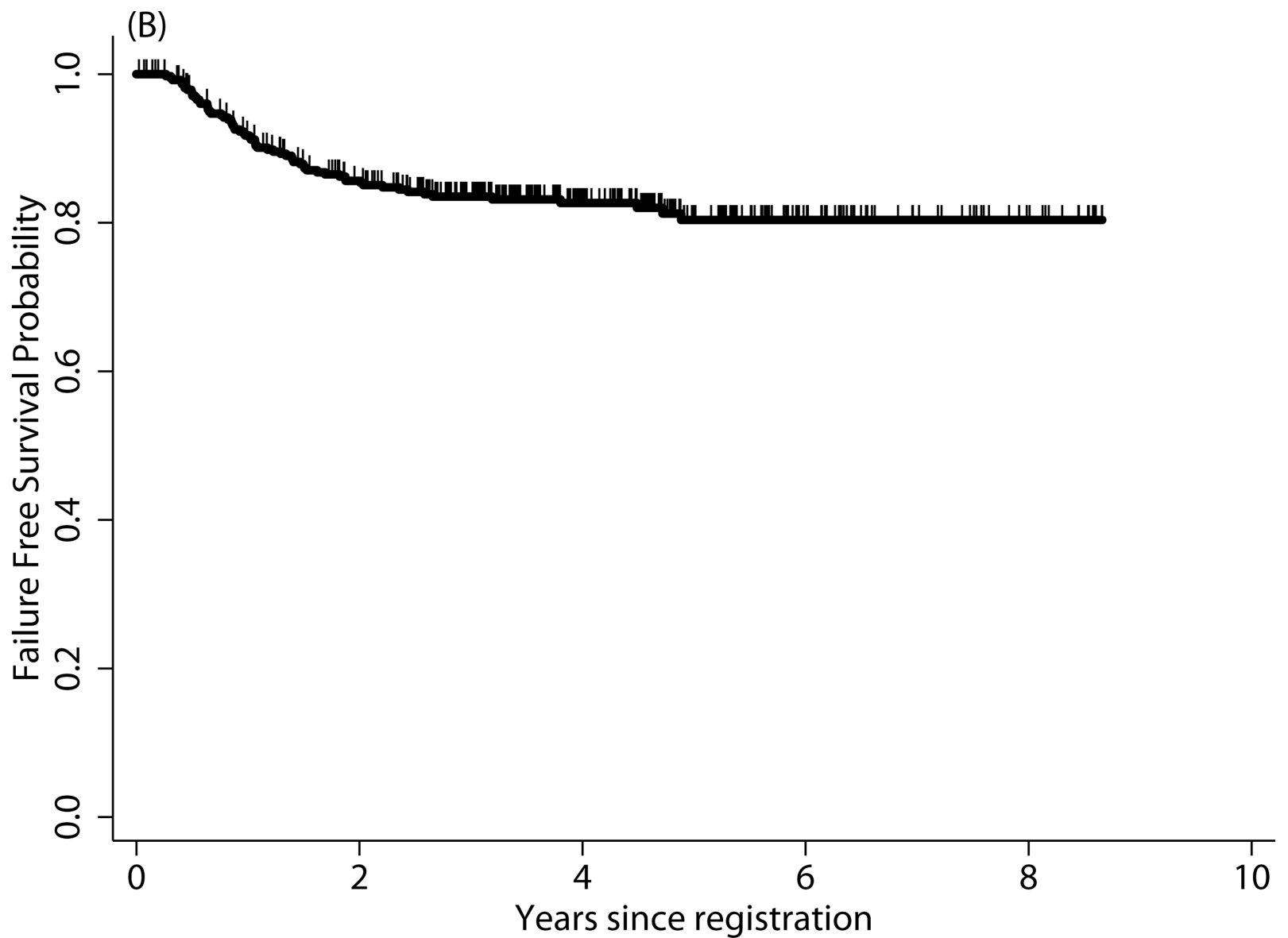

No. at Risk

FIGURE 1.

Failure-free survival (FFS) of Hodgkin lymphoma patients treated on E2496 (A) and AYA Hodgkin lymphoma patients treated on COG AHOD0031 (B); overall survival (OS) of Hodgkin lymphoma patients treated on E2496 (C) and AYA Hodgkin lymphoma patients treated on COG AHOD0031 (D) 
TABLE 1

Characteristics of AYA Patients Treated on E2496 and AHOD0031.

\begin{tabular}{|c|c|c|c|}
\hline & $\begin{array}{l}\text { AHOD0031 } \\
\text { AYAs } \\
\text { N=391 }\end{array}$ & $\begin{array}{l}\text { E2496 } \\
\text { AYAs } \\
\text { N=114 }\end{array}$ & P value ${ }^{*}$ \\
\hline \multicolumn{4}{|l|}{ Treatment } \\
\hline Induction only & $25(6.4 \%)$ & \multirow{8}{*}{$\begin{array}{l}56(49.1 \%) \\
58(50.9 \%)\end{array}$} & \\
\hline ABVE-PCX4, <CR, IFRT & $141(36.1 \%)$ & & \\
\hline ABVE-PCX4, CR, IFRT & $81(20.7 \%)$ & & \\
\hline ABVE-PCx4, CR, NO IFRT & $68(17.4 \%)$ & & \\
\hline ABVE-PCx4+IFRT+DECAx2 & $36(9.2 \%)$ & & \\
\hline ABVE-PCX4+IFRT & $40(10.2 \%)$ & & \\
\hline ABVD & & & \\
\hline Stanford V & & & \\
\hline Radiation received & $298(76.2 \%)$ & $75(65.8 \%)$ & 0.03 \\
\hline Male gender & $183(46.8 \%)$ & $59(51.8 \%)$ & 0.35 \\
\hline Mean age at enrollment (SD), years & $18.2(1.1)$ & $20.1(1.2)$ & $<0.001^{* * *}$ \\
\hline Range & $17.0-21.9$ & $17.2-21.9$ & \\
\hline Race/ethnicity & & & 0.07 \\
\hline Black & $38(10.2 \%)$ & $8(7.3 \%)$ & \\
\hline White & $279(75.0 \%)$ & $94(86.2 \%)$ & \\
\hline Hispanic & $39(10.5 \%)$ & $4(3.7 \%)$ & \\
\hline Other & $16(4.3 \%)$ & $3(2.8 \%)$ & \\
\hline Unknown & 19 & 5 & \\
\hline \multicolumn{4}{|l|}{ Stage } \\
\hline I & $25(6.4 \%)$ & $6(5.4 \%)$ & \multirow[t]{5}{*}{$<0.001^{\wedge}$} \\
\hline II & $253(64.7 \%)$ & $35(31.3 \%)$ & \\
\hline III & $61(15.6 \%)$ & $45(40.2 \%)$ & \\
\hline IV & $52(13.3 \%)$ & $26(23.2 \%)$ & \\
\hline Unknown & 0 & 2 & \\
\hline I+II & $278(71.1 \%)$ & $41(36.6 \%)$ & \multirow[t]{3}{*}{$<0.001^{\wedge}$} \\
\hline III & $61(15.6 \%)$ & $45(40.2 \%)$ & \\
\hline IV & $52(13.3 \%)$ & $26(23.2 \%)$ & \\
\hline Histology & & & $0.13^{\#}$ \\
\hline Lymphocyte predominant & $14(3.7 \%)$ & $1(1.0 \%)$ & \\
\hline Lymphocyte depleted & $1(0.3 \%)$ & $0(0 \%)$ & \\
\hline Nodular sclerosis & $339(90.4 \%)$ & $89(88.1 \%)$ & \\
\hline Mixed cellularity & $21(5.6 \%)$ & $11(10.9 \%)$ & \\
\hline Unknown & 16 & 13 & \\
\hline
\end{tabular}




\begin{tabular}{|c|c|c|c|}
\hline & $\begin{array}{l}\text { AHOD0031 } \\
\text { AYAs } \\
\text { N=391 }\end{array}$ & $\begin{array}{l}\text { E2496 } \\
\text { AYAs } \\
\text { N=114 }\end{array}$ & P value ${ }^{*}$ \\
\hline B symptoms & & & $<0.001$ \\
\hline No & $282(72.7 \%)$ & $41(36.6 \%)$ & \\
\hline Yes & $106(27.3 \%)$ & $71(63.4 \%)$ & \\
\hline Unknown & 3 & 2 & \\
\hline Bulk disease & & & $<0.001^{\# \#}$ \\
\hline No & $89(23.1 \%)$ & $76(66.7 \%)$ & \\
\hline Yes & $296(76.9 \%)$ & $38(33.3 \%)$ & \\
\hline Unknown & 6 & 0 & \\
\hline Extralymphatic disease $\mathrm{e}^{\# \# \#}$ & & & 0.24 \\
\hline No & $280(76.5 \%)$ & $81(71.1 \%)$ & \\
\hline Yes & $86(23.5 \%)$ & $33(28.9 \%)$ & \\
\hline Unknown & 25 & 0 & \\
\hline Anemia $($ Hgb $<10.5$ g/dl $)$ & & & 0.75 \\
\hline No & $310(82.0 \%)$ & $95(83.3 \%)$ & \\
\hline Yes & $68(18.0 \%)$ & $19(16.7 \%)$ & \\
\hline Unknown & 13 & 0 & \\
\hline Albumin $<4$ g/dl & & & 0.11 \\
\hline No & $149(40.0 \%)$ & $36(31.6 \%)$ & \\
\hline Yes & $224(60.0 \%)$ & $78(68.4 \%)$ & \\
\hline Unknown & 18 & 0 & \\
\hline
\end{tabular}

P values from chi-square tests unless otherwise specified

**

t-test;

Wilcoxon rank-sum test;

\# Fisher's exact test

\#\# This difference in the proportion with bulk disease between the child and adult cohort was only seen among Stage III and IV patients, not among Stage I or II patients: Stage I - 96\% child, $100 \%$ adult; Stage II - 84\% child, $86 \%$ adult; Stage III - 59\% child, $0 \%$ adult; Stage IV - 55\% child, 0\% adult

\#\#\# Extralymphatic disease refers to extranodal extension 
TABLE 2

Planned cumulative chemotherapy exposures by regimen.

\begin{tabular}{|l|l|l|l|l|}
\hline & $\begin{array}{l}\text { ABVD } \\
\text { (6-8 cycles; } \\
\text { 24-32 weeks) }\end{array}$ & $\begin{array}{l}\text { Stanford V } \\
\text { (12 weeks) }\end{array}$ & $\begin{array}{l}\text { ABVE-PC } \\
\text { (4 cycles; } \\
\text { 12 weeks) }\end{array}$ & $\begin{array}{l}\text { DECA } \\
\text { (2 cycles; 6 } \\
\text { weeks })\end{array}$ \\
\hline & \multicolumn{4}{|c|}{ Planned cumulative exposure } \\
\hline Doxorubicin & $300-400 \mathrm{mg} / \mathrm{m}^{2}$ & $150 \mathrm{mg} / \mathrm{m}^{2}$ & $200 \mathrm{mg} / \mathrm{m}^{2}$ & --- \\
\hline Bleomycin & $120-180 \mathrm{U} / \mathrm{m}^{2}$ & $30 \mathrm{U} / \mathrm{m}^{2}$ & $60 \mathrm{U} / \mathrm{m}^{2}$ & --- \\
\hline Vinblastine & $72-96 \mathrm{mg} / \mathrm{m}^{2}$ & $36 \mathrm{mg} / \mathrm{m}^{2}$ & --- & --- \\
\hline Vincristine & --- & $8.4 \mathrm{mg} / \mathrm{m}^{2}$ & $11.2 \mathrm{mg} / \mathrm{m}^{2}$ & --- \\
\hline Dacarbazine & $4.5-6.0 \mathrm{~g} / \mathrm{m}^{2}$ & --- & --- & --- \\
\hline Cyclophosphamide & --- & --- & $3.2 \mathrm{~g} / \mathrm{m}^{2}$ & --- \\
\hline Mechlorethamine & --- & $18 \mathrm{mg} / \mathrm{m}^{2}$ & --- & --- \\
\hline Etoposide & --- & $360 \mathrm{mg} / \mathrm{m}^{2}$ & $1,500 \mathrm{mg} / \mathrm{m}^{2}$ & $400 \mathrm{mg} / \mathrm{m}^{2}$ \\
\hline Prednisone & --- & $\sim 420 \mathrm{mg} / \mathrm{m}^{2 *}$ & $1,120 \mathrm{mg} / \mathrm{m}^{2}$ & --- \\
\hline Dexamethasone & --- & --- & --- & $40 \mathrm{mg} / \mathrm{m}^{2}$ \\
\hline Cytarabine & --- & --- & --- & $12 \mathrm{~g} / \mathrm{m}^{2}$ \\
\hline Cisplatin & --- & --- & --- & $180 \mathrm{mg} / \mathrm{m}^{2}$ \\
\hline
\end{tabular}

* Cumulative dose does not reflect taper in weeks 10-12. 


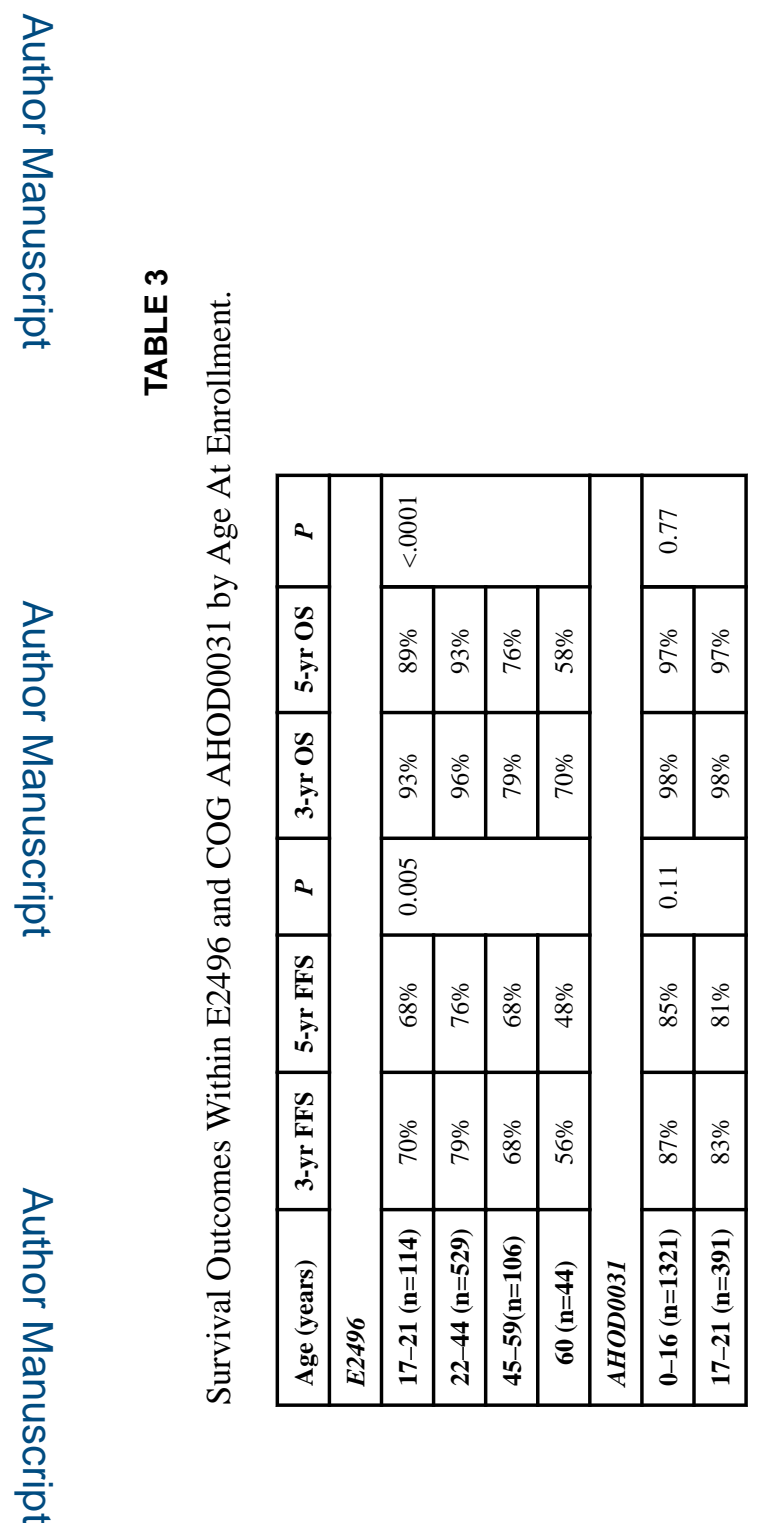

Cancer. Author manuscript; available in PMC 2019 January 01. 